\title{
Máquinas Nomológicas, Modelos e Intervención: su Problemática en el Campo de lo Social ${ }^{1}$
}

\author{
Leonardo Ivarola ${ }^{2}$
}

\begin{abstract}
RESUMEN: En el presente trabajo se hará una crítica al enfoque de máquinas nomológicas desarrollado por Nancy Cartwright para el caso de las ciencias sociales. Se argumentará que los fenómenos sociales no responden a una lógica de "capacidades", sino de "árboles de posibilidades" o "resultados de final abierto". La estabilidad o invarianza, si bien es posible en el reino de lo social, parece ser más la excepción que la regla. Esto tiene consecuencias importantes para los propósitos de intervención y control. De acuerdo con las corrientes manipulabilistas tradicionales la invarianza es una herramienta fundamental para cumplir cualquier propósito intervencionista, ya que permite prever con un buen grado de confianza el resultado de una manipulación. Sin embargo, en este trabajo se intentará mostrar que, a pesar de que no sea del todo plausible obtener un conocimiento invariante en el reino de lo social, las intervenciones pueden ser igualmente eficaces. Para ello, será fundamental reemplazar la noción "ex-ante" de intervención por una que involucre continuidad o sistematicidad. También será clave tener en cuenta aquellas cláusulas o condiciones especificadas dentro de los modelos, ya que estos últimos cumplen el rol de ser anteproyectos para la generación de resultados.
\end{abstract}

PALABRAS CLAVE: Máquinas nomológicas. Intervención. Resultados de final abierto. Capacidades sociales.

\section{INTRODUCCión}

De acuerdo con Nancy Cartwright, la ciencia procura descubrir las "capacidades" o naturalezas de las cosas. Las regularidades son secundarias. En todo caso, éstas emergen de circunstancias muy específicas donde en un sistema particular de componentes - denominado "máquina nomológica" operan dichas capacidades de manera repetida. Así, las capacidades resultan ser más fundamentales que las regularidades o leyes, puesto que las últimas siempre se obtienen a causa de las primeras (HÉDOIN, 2012).

Las máquinas nomológicas son cruciales para el armado el implementación de políticas, ya que a través de su ejercicio se obtiene un conocimiento invariante, y la invarianza es una condición necesaria para

\footnotetext{
${ }^{1}$ http://dx.doi.org/10.1590/S0101-31732016000200006

${ }^{2}$ Universidad de Buenos Aires. E-mail: ivarola@economicas.uba.ar
} 
satisfacer propósitos intervencionistas (WOODWARD, 2002, 2003; CARTWRIGHT, 2009b; REISS, 2007). En la medida en que en el mundo real se cumplan todas las condiciones especificadas dentro de un modelo, cualquier manipulación sobre una máquina nomológica estará garantizada, en el sentido de que los valores predichos dentro de dicho modelo se corresponderán con los valores reales. Esto es así ya que las máquinas nomológicas son sistemas cerrados conformados por capacidades estables y apropiadamente protegidos de cualquier influencia externa (CARTWRIGHT, 1999a).

Si bien esta concepción puede ser útil para las ciencias naturales, no parece serlo de igual manera para las ciencias sociales. En estas últimas no prevalecen las capacidades, sino los "resultados de final abierto" o "árboles de posibilidades": no existe una conducta predeterminada por parte de las personas, sino que éstas pueden tomar distintas decisiones de acuerdo a cómo formen sus expectativas respecto de ciertos eventos futuros, de cómo interpreten las señales del mundo, de cómo su accionar se vea restringido por factores institucionales, etc. Si esto es cierto, entonces no parece plausible la construcción de máquinas nomológicas en los sistemas sociales. Tampoco parece viable requerir la obtención de un conocimiento invariante para propósitos de política. Por un lado, porque existen incompatibilidades a nivel ontológico: no hay contribuciones estables, sino resultados de final abierto. Por el otro lado, porque incluso obteniendo dichas contribuciones estables, nada garantiza que en los periodos subsiguientes esta estabilidad prevalezca.

Sin embargo, que las máquinas nomológicas no sean plausibles en el reino de lo social - y en general, que las regularidades invariantes sean más la excepción que la regla - no significa que no se puedan implementar políticas. En este sentido, el presente trabajo tiene como objetivo reflexionar, por un lado, acerca de la clase de conocimiento que se puede utilizar para propósitos de implementación de políticas, y por el otro, acerca de las formas en que se llevan a cabo las intervenciones. En primer lugar, se argumentará que el conocimiento proporcionado por los modelos no refiere a factores causales aislados (MÄKI, 1992; CARTWRIGHT, 1998; HÉDOIN, 2012), sino a anteproyectos donde se especifican el acervo de condiciones que acotan el rango de posibilidades de un sistema social a fin de llegar al resultado deseado. También se tratará de arrojar luz sobre la noción de intervención. En los enfoques manipulabilistas tradicionales en general (WOODWARD, 2000, 2002, 2003; CARTWRIGHT, 2012; CARTWRIGHT; HARDIE, 2013; HENDRY, 2004; MITCHELL, 1997, 2003; REISS, 2007) y en el de 
máquinas nomológicas en particular (CARTWRIGHT, 1995, 1997, 1999a, 2009b) se asume que las intervenciones siempre son ex-ante: se generan un conjunto de condiciones antecedentes, se activa el factor disparador, y se espera a que el resultado llegue de manera automática. Por el contrario, en el presente trabajo se argumentará que las intervenciones en el reino de lo social deben ser sistemáticas, ya que no hay ningún resultado garantizado de antemano. Por un lado, dichas intervenciones apuntarán a satisfacer las condiciones explicitadas en el anteproyecto. Por el otro, se intentarán corregir los posibles desvíos generados por cambios en la toma de decisiones de las personas. A diferencia del primer tipo de intervención mencionado, este último involucra una noción de "sistematicidad" o "continuidad".

\section{CAPACIDADES Y MÁQUINAS NOMOLÓGICAS}

En The Dappled World: A Study of the Boundaries of Science (1999a), Nancy Cartwright dirige su crítica al enfoque "legalista" de la ciencia. Según este enfoque, el mundo real es un mundo ordenado donde reinan las leyes, las cuales son comprendidas como conjunciones constantes de eventos. Éste es el legado de aquellos filósofos de la ciencia que toman a la física como modelo a seguir. Cartwright se opone a esta visión de la ciencia. Para la autora, buena parte de lo que ocurre en la naturaleza ocurre por casualidad, sujeto a ningún tipo de ley. El mundo es "desordenado". No hay ninguna garantía que una regularidad que prevalece hoy se mantenga también mañana. Contrario a ello, la autora defiende la idea de que las afirmaciones causales de la ciencia no son acerca de regularidades o conjunciones constantes de eventos, sino acerca de capacidades que subyacen a dichas regularidades.

Básicamente, las capacidades son propiedades de entidades y/o variables que contribuyen a la producción de un resultado. Dicha contribución no debe ser entendida en términos legaliformes. Cuando se asevera que "[...] la aspirina tiene la capacidad de aliviar el dolor de cabeza", lo que se está diciendo es que existe una entidad con la propiedad de producir un resultado. No hay un compromiso "legal" del tipo "[...] siempre que se tome una aspirina se aliviará el dolor del cabeza”, ni tampoco que alivie la mayor parte de las veces. En lugar de ello, lo que simplemente se dice es que existe una capacidad estable y relativamente duradera que una entidad lleva consigo misma de caso en caso (CARTWRIGHT, 1989). 
El concepto de "capacidad" descansa en una distinción tripartita (CARTWRIGHT, 1989): (1) la existencia/obtención de una capacidad, (2) su ejercicio, y (3) sus resultados manifiestos. Tomemos como ejemplo de esto la noción de gravedad. La gravedad es la capacidad que tienen los cuerpos de ser atraídos mutuamente. Dicha capacidad es una contribución estable que ya existe en el universo. Ahora bien, en el supuesto caso de que en un sistema no se dé la presencia de al menos dos objetos, esta capacidad puede no ejercitarse nunca. También existe la posibilidad de que, ejercitándose dicha capacidad, los resultados no se manifiesten (por ejemplo, si existe un tercer cuerpo que ejerza una fuerza opuesta). La contribución de la capacidad es estable. Empero, de ello no se sigue que ésta vaya a ser directamente observable al nivel de los eventos.

Las capacidades son los elementos primitivos del mundo. Las regularidades son secundarias. En tal caso, dichas regularidades son una consecuencia de la operación repetida de estructuras o mecanismos que tienen capacidades estables, organizadas "correctamente" en el medio "correcto" (CARTWRIGHT, 1995). La imagen de esto es una máquina blindada o protegida de influencias externas. Esto permite que el conjunto de componentes ensamblados dentro de la máquina funcione sin perturbaciones. $\mathrm{Y}$ antes de esperar cualquier patrón de regularidad entre inputs y outputs, dicha máquina deberá ser puesta en funcionamiento numerosas veces. Así, las regularidades son una consecuencia de la operación satisfactoriamente repetida de una máquina nomológica, la cual define como

[...] a fixed (enough) arrangement of components, or factors, with stable (enough) capacities that in the right sort of stable (enough) environment will, with repeated operation, give rise to the kind of regular behavior that we represent in our scientific laws. (CARTWRIGHT, 1999a, p. 50).

Un buen ejemplo de lo que es una máquina nomológica lo proporcionan las conocidas máquinas expendedoras de gaseosas o de golosinas. Se trata de una máquina cuyo funcionamiento repetido da lugar a una regularidad del tipo "input - output" (inserción de moneda - retiro del producto). Este proceso comienza cuando se inserta la moneda y se oprime el botón correspondiente al producto deseado. En el interior de la máquina ocurren una serie de procesos mecánicos que culminan con la obtención del producto seleccionado. La regularidad emerge del funcionamiento satisfactoriamente repetido de la máquina: (casi) siempre que suceda $\mathrm{x}$ (se inserte la moneda), ocurrirá $\mathrm{y}$ 
(obtención del producto). Ahora bien, para que esto sea así, la máquina debe estar aislada de cualquier factor que pueda perturbar su buen funcionamiento. Esto es precisamente lo que ocurre con una máquina expendedora de gaseosas: el mecanismo está protegido de varios (aunque posiblemente no todos) tipos de influencias externas.

Puesto que las máquinas nomológicas proporcionan un conocimiento invariante, éstas resultan ser invaluables para el diseño e implementación de políticas (véase CARTWRIGHT, 2009b). De acuerdo con las corrientes "manipulabilistas" tradicionales (WOODWARD, 2000, 2002, 2003; CARTWRIGHT，2009b， 2012; CARTWRIGHT; HARDIE， 2013; HENDRY, 2004; MITCHELL, 1997, 2003; REISS, 2007), una correcta implementación de política debe estar sustentada en un conocimiento factores estables o invariantes. La invarianza es una condición necesaria para esta clase de propósitos, ya que lo que se desea es conocer cuál será el resultado de manipular un determinado factor o conjunto de factores, y sin invarianza dicha predicción sería prácticamente imposible.

El conocimiento proporcionado por las máquinas nomológicas es un conocimiento sobre regularidades ceteris paribus. Estas cláusulas son condiciones de importancia capital en el surgimiento de regularidades, ya que "[...] describen la estructura de la máquina que hace que esa regularidad sea verdadera." (CARTWRIGHT, 1995, p.289). Esto tiene un punto favorable y uno desfavorable en lo concerniente a los propósitos de manipulación y control. El punto favorable está asociado a la idea de que, en tanto estas condiciones se satisfagan en el sistema objetivo, las intervenciones van a ser exitosas, ya que en éste emergerá una regularidad invariante. Por su parte, el punto desfavorable está asociado con un problema de validez externa. Se tiene validez externa cuando el resultado de una política, experimento, etc., se mantiene fuera de su respectivo dominio. No obstante, el descubrimiento de una relación invariante en ausencia de un conjunto factores perturbadores no nos asegura que ésta prevalezca una vez que dichos factores estén presentes (CARTWRIGHT, 2007). Esto significa que las máquinas nomológicas proporcionan mediante su ejercicio repetitivo el conocimiento de regularidades invariantes. Sin embargo, éstas valen para dominios muy acotados, por lo cual cualquier política que se desee implementar fuera del dominio de las máquinas nomológicas no estará garantizada (CARTWRIGHT, 2009a, 2009b). 


\section{MOdelos DE MÁQUiNAS NOMOLÓGICAS: ¿REPRESENTACIONES AISLADAS O ANTEPROYECTOS PARA LA CONSTRUCCIÓN DE SISTEMAS COMPLEJOS?}

De acuerdo con Cartwright (1995), los modelos en ciencias sociales ${ }^{3}$ no son construcciones edificadas sobre la base de regularidades fundamentales de las cuales el modelador puede deducir algún caso especial. Los modelos son anteproyectos de máquinas nomológicas. En estos se explicitan todas las condiciones que se necesitan cumplir en el mundo real para que el resultado inferido dentro del modelo se vea reflejado a nivel empírico.

Ahora bien, de acuerdo a cómo se conciba la ontología de las máquinas nomológicas, distinta será la concepción respecto del rol de los modelos que las sustentan. En particular, hay dos maneras de entender a las máquinas nomológicas: como factores causales donde se aísla la contribución de un mecanismo o capacidad, y como un sistema complejo dotado por un conjunto de capacidades cuya interacción da lugar a un comportamiento regular. Dada esta distinción ontológica podemos concebir a los modelos de máquinas nomológicas de la siguiente manera: (1) como aislamientos teoréticos, y (2) como anteproyectos o bocetos para la construcción de sistemas complejos que generan resultados.

Con respecto a (1), el método de aislamiento teorético consiste en separar un factor causal de cualquier elemento perturbador, de modo tal que dicho factor pueda encontrarse en "estado puro". En estos casos, el rol de las cláusulas ceteris paribus consistiría en neutralizar la presencia de factores perturbadores, sea ya anulando su contribución como blindando al sistema para impedir su posible presencia (CARTWRIGHT, 1998; MÄKI, 1992, HÉDOIN, 2012). A diferencia de los experimentos de laboratorio, el aislamiento teorético no tiene lugar en un sistema concreto o real, sino en uno conceptual. ${ }^{4}$ Sin embargo, Mäki (1992, 2005) considera que el aislamiento teorético es una operación intelectual de características semejantes a la experimentación científica: ambas involucran un proceso de intervención a través de la clausura de posibles factores perturbadores. Por esta razón, Mäki concibe a las aislaciones teoréticas como experimentos mentales. Los aislamientos se logran a través de la manipulación de ciertas entidades. Lo que diferencia un experimento mental de uno material depende de qué elemento ha de ser manipulado.

\footnotetext{
${ }^{3}$ Cartwright (1995) enfatiza el caso de los modelos en economía.

${ }^{4}$ La distinción entre sistema conceptual y concreto ha sido tomada de Bunge (2000).
} 
El segundo punto concibe a las máquinas nomológicas como sistemas complejos. Al respecto, Cartwright asevera que algunas veces las máquinas nomológicas existen en la naturaleza sin intervención alguna de la mano del hombre $(v . g r$., el sistema solar). No obstante, en numerosas situaciones es esta acción la que debe ponerse en práctica, a los efectos de lograr un comportamiento estable al nivel de los eventos. En casos como estos, la máquina nomológica puede ser entendida como un sistema complejo altamente estructurado de capacidades estables construida con el propósito de producir un resultado regular. Dicha construcción está sustentada en un anteproyecto que especifica un conjunto de capacidades sociales y de cláusulas ceteris paribus. A diferencia del caso anterior, estas cláusulas no se corresponden (al menos no necesariamente) con neutralizaciones de factores causales, sino más bien con condicionamientos referidos a la estructura que se le impone a un sistema a fin de lograr el resultado buscado. En otras palabras, los supuestos de esta clase de modelos no cumplirían una función de "neutralización", sino la de proporcionar una cierta estructura que facilite la inferencia de resultados dentro de los mismos (CARTWRIGHT, 2009a).

Dadas estas dos concepciones de máquinas nomológicas se pueden pensar en dos clases de intervenciones: (1) como manipulaciones dirigidas a la activación de factores disparadores, y (2) como manipulaciones que tanto activan factores disparadores como participan en la propia conformación de la máquina.

La primera clase de intervención es la que se utiliza comúnmente en los enfoques manipulabilistas modernos: dada una capacidad, mecanismo, regularidad causal invariante, etc., en cada una de ellas la variable intervención cumple el rol en activar el factor que dispara su funcionamiento.

La segunda clase no sólo involucra a la primera, sino que también refiere a un aspecto "tecnológico". Específicamente hablando, la intervención no sólo consiste en activar el factor disparador de la máquina, sino que también participaría en la conformación de la misma. La máquina expendedora de gaseosas es un buen ejemplo de ello: no sólo se debe activar algún botón de ésta para seleccionar el producto; la propia construcción de la máquina es, en sí misma, una intervención.

Como se puede apreciar, bajo este modo de pensamiento la única clase de intervención es "ex-ante", es decir, en las condiciones de inicio o de set up, sean ya participando en la configuración de los elementos constituyentes de 
una máquina como en la activación de sus respectivos factores disparadores. Una vez hechas estas intervenciones, la máquina funcionará de manera automática, sin la necesidad de realizar nuevas intervenciones.

\section{LOS FENÓMENOS SOCIALES COMO RESULTADOS DE FINAL ABIERTO}

El enfoque de máquinas nomológicas desarrollado por Cartwright presupone la existencia de factores estables o invariantes. Este supuesto no parece ser problemático para las ciencias naturales, o al menos para aquellas ciencias en las cuales las acciones de las personas no juegan un rol causal sustantivo. Cuando decimos que "la aspirina tiene la capacidad de aliviar el dolor de cabeza”, en realidad estamos hablando de una contribución estable o invariante de una entidad en la producción de un resultado, producción que es funcional a las propiedades de la entidad participante (la aspirina) y del sistema en donde se hace la manipulación (el cuerpo humano).

No obstante, dicha suposición sí es problemática en el reino de lo social. Específicamente, los fenómenos que tienen lugar en un sistema social responden a la lógica de los "árboles de posibilidades" o "resultados de final abierto": dado un determinado evento que funcione como factor disparador, distintas alternativas son posibles. Aquella que prevalezca será producto de las acciones que hayan tomado las personas, las cuales no están predeterminadas ex-ante. Así, por ejemplo, sea $X$ la variable independiente (o causa), $Y$ la variable dependiente (o efecto), y $A$ la acción o actividad de las personas. Supongamos que $X$ toma un valor $X_{0}$. Por definición, la variable $Y$ podrá tomar más de un valor $\left(Y_{1}\right.$, $\left.Y_{2}, \ldots, Y_{\mathrm{n}}\right)$. Todo dependerá de las acciones $A_{1}, A_{2}, \ldots, A_{\mathrm{n}}$ de las personas.

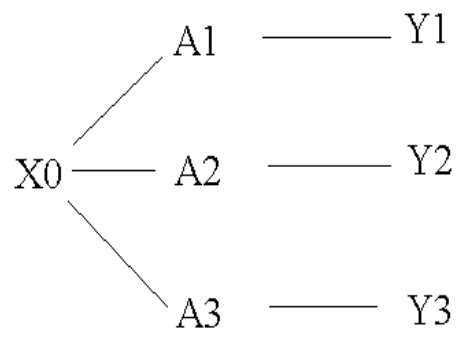

Un buen ejemplo de esto es lo que en economía se conoce como el "efecto Keynes". Éste es un fenómeno por el cual un aumento en la cantidad real 
de dinero provoca un incremento en la renta nacional. En particular, cuando aumenta la cantidad de dinero se acrecienta la demanda de activos financieros. Dicho acrecentamiento hace descender la tasa de interés. Por su parte, una tasa de interés más baja vuelve a los créditos más baratos, estimulando así la inversión. Finalmente, una mayor demanda de inversión trae consigo un aumento en la demanda de empleo que, de efectivizarse, provocaría tanto un descenso en el nivel de paro como un aumento en la renta nacional.

En algunas interpretaciones se asume que este cambio en la cantidad de dinero conducirá de manera automática e invariante a un cambio inverso en la tasa de interés; ídem con el resto de la secuencia. Sin embargo, esto no es así. Por el contrario, de acuerdo con el marco contextual y con las interpretaciones y expectativas que formen las personas, distintos serán los caminos que pueda tomar esta clase de procesos. Consciente de estas limitaciones, Keynes (1936, p.150) comenta:

[...] si bien puede esperarse que, ceteris paribus, un aumento en la cantidad de dinero reduzca la tasa de interés, esto no sucederá si las preferencias por la liquidez del público aumentan más que la cantidad de dinero; y mientras que puede esperarse que, ceteris paribus, un descenso en la tasa de interés aumente el volumen de la inversión, esto no ocurrirá si la curva de la eficiencia marginal del capital baja con mayor rapidez que la tasa de interés; y mientras es de suponer que, ceteris paribus, un aumento en el volumen de la inversión haga subir la ocupación, esto puede no suceder si la propensión marginal a consumir va en descenso.

En los sistemas naturales, los mecanismos se comportan de manera estable justamente porque las actividades que se llevan a cabo dentro de los mismos son estables. Por ejemplo, en el mecanismo de neurotransmisión química una neurona pre-sináptica trasmite una señal a una neurona post-sináptica a través de la liberación de moléculas neurotransmisoras que se propagan a través de la hendidura sináptica, se enlazan con los receptores, y así la célula post-sináptica es despolarizada. Usando la terminología de Machamer, Darden y Craver (2000), 5 el neurotransmisor y el receptor son dos entidades, mientras que el acto de enlace es una actividad que se lleva a cabo en virtud de sus propiedades estructurales y de sus distribuciones de carga. Este mecanismo se comporta de manera regular

\footnotetext{
${ }^{5}$ Machamer, Darden y Craver (2000, p. 3) definen a los mecanismos como "[...] entidades y actividades organizadas de tal manera que son productoras de cambios regulares, desde las condiciones de inicio o set-up a las condiciones de finalización o terminación”.
} 
precisamente porque la actividad central que se lleva a cabo dentro del mismo el acto de enlace - es estable o invariante.

En contraste, las actividades que se llevan a cabo en el interior de los procesos sociales se corresponden con las acciones de las personas, las cuales pueden ser muy volátiles. Por ejemplo, cualquier cambio en la interpretación de las señales recibidas podrá redundar en una importante modificación en el proceso de formación de expectativas; cualquier cambio institucional podrá re-direccionar los cursos de acción de las personas, etc. Esta observación pone en tela de juicio la tesis respecto de la existencia de contribuciones estables en el reino de lo social - esto es, de "capacidades sociales" -, justamente porque no existe una estabilidad per se de las actividades de las personas. Más aun, de ser cierto esto, entonces el enfoque de máquinas nomológicas también se desmorona, en tanto y en cuanto para el armado de las mismas se requiere de la disposición de factores estables.

Sin embargo, el problema no termina allí. En la construcción de una máquina nomológica se requiere que sus elementos constituyentes estén correctamente configurados unos con otros, y protegidos de cualquier influencia externa, a los efectos de que (casi) siempre que se haga $X$, se obtenga el resultado $Y$.

Supongamos por un momento que esta configuración fuese posible - en otras palabras, que en la investigación social haya sido posible el descubrimiento de determinadas capacidades sociales-. El problema surgirá cuanto se intente proteger a la máquina de influencias externas. En una máquina expendedora de gaseosas el mecanismo interno está protegido de numerosos tipos de manipulaciones, sean ya humanos como de la naturaleza. Esto permite que la misma se comporte de manera regular, en el sentido de que (casi) siempre que se introduzca una(s) moneda(s) en la ranura, la máquina proporcione la gaseosa seleccionada previamente por el comprador. ¿Es esto posible en los sistemas sociales? ¿Podemos blindar mecanismos sociales en el mundo real?

La tarea parece, más que compleja, imposible, ya que en los procesos sociales cualquier factor exógeno puede perturbar el buen funcionamiento de la supuesta máquina. Si bien uno puede esperar controlar algunas variables en un sistema social, no parece sensato pretender controlarlas todas. En los sistemas sociales, algunas de sus variables constituyentes no son conocidas de antemano (LAWSON, 1997; DOW, 1998). Esta incertidumbre hace 
que en numerosas situaciones no sepamos a ciencia cierta qué va a suceder en el futuro próximo. Podemos prever con cierta confianza la ocurrencia de algunos acontecimientos, pero no hay garantía de ello. Y con respecto a otros hechos, ni siquiera pensamos acerca de su posibilidad fáctica. Si no sabemos qué circunstancias acontecerán, entonces tampoco parece adecuado pretender blindar o proteger un mecanismo de las mismas.

Incluso suponiendo que este blindaje fuese viable, existe la posibilidad de que una máquina nomológica proporcione resultados inestables a causa de problemas endógenos. Volviendo con el ejemplo del efecto Keynes, éste puede ser considerado como un "mecanismo" que prevalece en la medida en que ciertas condiciones antecedentes se cumplan. Supongamos que se ha hecho todo lo posible para que ese mecanismo funcione en el mundo real $(v$. gr., arreglos institucionales, buenas expectativas, etc.), y que por tal razón la autoridad monetaria contribuya al crecimiento económico vía aumento en la cantidad real de dinero. Sin embargo, supongamos que, en algún momento, un empresario empezase a desconfiar acerca de la posibilidad de vender todos sus bienes en el próximo periodo, y que a causa de ello éste decida reducir su nivel de inversión. Supongamos adicionalmente que esta estrategia se empieza a propagar entre los empresarios allegados a éste (mecanismo conocido como “comportamiento de manada”). Cuanto mayor sea esta propagación, mayor será el efecto negativo sobre el empleo y la producción. Esta inestabilidad no fue producto de fallas en el blindaje o en el ensamble de partes. La misma tiene su origen en un problema "endógeno".

Ahora bien, lo anterior no implica que no sea posible observar secuencias regulares al nivel de los eventos. No sólo son plausibles, sino que la historia da cuenta de su factibilidad. Sin embargo, esto es posible no porque sean producto de mecanismos "transfácticos" (LAWSON, 1997, 2003) operando en los sistemas sociales o por el ejercicio de contribuciones que por naturaleza son estables, sino a causa de una conformación de expectativas poco volátiles, de arreglos institucionales estables en el tiempo, etc.

Tomemos como ejemplo de ello la famosa "curva de Phillips". El economista William Phillips publicó en 1958 un artículo titulado "La relación entre el desempleo y la tasa de variación de los salarios monetarios en el Reino Unido, 1861-1957”, en el cual se establecía la existencia de una correlación negativa entre la tasa de desempleo y la inflación. Dos años más tarde, Samuelson y Solow encontraron el mismo registro estadístico para Estados Unidos entre 1900 y 1960. La explicación de esta correlación inversa entre 
inflación y desempleo es sencilla: dados los precios esperados, que para los trabajadores son simplemente los precios del año anterior, una reducción del desempleo provoca una subida de los salarios nominales, la cual provoca, a su vez, una subida de los precios.

No obstante, esta regularidad no se mantuvo entre 1960 y 1970 . El proceso inflacionario se acentuó durante estos años, lo cual modificó el modo en el que las personas formaban sus expectativas. Durante los periodos de inflación baja y poco persistente, tanto los trabajadores como las empresas solían no tener en cuenta la inflación pasada, suponiendo así que los precios en los próximos periodos no iban a diferir significativamente de los precios actuales. Pero en un marco de inflación sistemática, los agentes empezaron a suponer que en los próximos periodos los precios seguirían aumentando, haciendo que la relación inversa entre inflación y desempleo desaparezca. El ejemplo muestra que de haber estabilidad en ciertas condiciones antecedentes en este caso, de expectativas de inflación baja - una regularidad puede emerger al nivel de los eventos. No obstante, ésta no es producto de contribuciones estables como las capacidades, sino de un conjunto de circunstancias específicas de validez temporal limitada y sin ningún tipo de "blindaje". Cuando estas circunstancias o condiciones ya no se cumplan, la regularidad desaparecerá. Aquí no hay una compensación de factores causales estables (como sucede cuando el resultado manifiesto de una capacidad no emerge por la presencia de factores perturbadores); lo que hay es una nueva producción o contribución.

\section{Modelos E INTERVENCIÓN}

En el acápite 3 se comentó que los modelos de máquinas nomológicas podían ser entendidos de dos maneras diferentes: como aislamientos de mecanismos o como anteproyectos para la construcción de dichas máquinas.

El primer caso está asociado con el enfoque de modelos como aislamientos teoréticos. Este enfoque presupone una ontología de factores causales estables tales como los mecanismos (MÄKI, 1992, 2009, 2011) o las capacidades (CARTWRIGHT, 1998, 1999a). Sin embargo, es dudoso que este aislamiento sea posible en aquellas situaciones en donde lo que prevalece son los árboles de posibilidades en lugar de los mecanismos o las capacidades. De acuerdo con esta noción, no habría una secuencia predeterminada que conduzca de una causa $X$ hacia un efecto $Y$. Al activar $X$ podrá ocurrir $Y_{1}$, $Y_{2}, \ldots, Y_{\mathrm{n}}$. Para que cualquiera de estos resultados ocurra deberán cumplirse 
una serie de condiciones adicionales. No hay un proceso de "separación" o de "neutralización" de elementos perturbadores, sino de "adición" de nuevos factores condicionantes. ${ }^{6}$ El ejemplo del efecto Keynes mencionado anteriormente es claro: no se trata de un mecanismo por el cual si neutralizamos todos los factores perturbadores pasaremos de un estado inicial de aumento en la cantidad real de dinero a un estado final con aumento en la renta nacional. Para que esta secuencia sea plausible, varias condiciones deberán cumplirse: que la tasa de interés esté en sus valores "normales", que los empresarios tengan buenas expectativas de ventas futuras, que las familias no disminuyan su propensión marginal a consumir, que haya confianza en la moneda que se está emitiendo, etc. De no darse cualquiera de estas restricciones, la secuencia se desviará por otro camino y el resultado esperado nunca aparecerá.

El conocimiento proporcionado por los modelos no es entonces un conocimiento de factores causales estables operando en aislamiento. Contrario a ello, los modelos nos brindan información sobre un conjunto de condiciones que, de satisfacerse en el mundo real, permitiría la aparición de determinados resultados (que son las inferencias del modelo). No es que exista en el mundo real una capacidad social que diga que el aumento en la oferta monetaria conlleva a un incremento en el nivel de renta nacional. Por el contrario, ciertas condiciones tienen que cumplirse para que ello se dé así y no de otra manera. Esta postura - más "tecnológica” - está acorde con la segunda interpretación de los modelos de máquinas nomológicas: los modelos como anteproyectos para la generación de resultados. De acuerdo con este punto de vista, los resultados no emergen del ejercicio aislado de contribuciones estables, sino de una construcción previa. Asimismo, la manipulación en el mundo real no sólo concierne a la activación de factores disparadores, sino también a la propia construcción de la máquina. El modelo no representa un mecanismo aislado; representa un anteproyecto donde se explicita el acervo de condiciones que se deberá satisfacer en el mundo real para alcanzar el resultado deseado.

Ahora bien, es importante tener en cuenta que dicho cumplimiento no garantiza la aparición de resultados en el mundo real. Esto se debe a que, como se comentó anteriormente, cualquier elemento exógeno no previsto puede alterar el sendero buscado hacia otros nodos del árbol de posibilidades. Por consiguiente, la noción de intervención mencionada en el acápite 3 debe ser replanteada. Según se ha visto, trátese de modelos como representaciones de factores causales aislados o como anteproyectos para la construcción de

${ }^{6}$ Una tesis similar es planteada en Cartwright (2009a). 
sistemas complejos, en cualquiera de los casos las intervenciones refieren a una noción "ex-ante". Esta es la postura de los enfoques manipulabilistas tradicionales, quienes al asumir un conocimiento previo de factores causales estables sostienen que las intervenciones deben ser siempre ex-ante. Es esta clase de supuestos la que lleva a dichos enfoques a establecer analogías de intervenciones sobre entidades como las máquinas expendedoras de gaseosas. Pero si los procesos sociales no son producto de factores causales estables, entonces la analogía no debe buscarse con las máquinas, sino con casos que involucren intervenciones sistemáticas, como el conducir un automóvil o el dirigir un partido de fútbol. Al conducir un automóvil uno debe intervenir permanentemente. Por más que tenga un GPS, sepa cómo pasar los cambios, haya revisado la presión de las cubiertas, el aceite, etc., el conductor debe estar atento todo el viaje, haciendo las intervenciones oportunas que le permitan llegar al destino. El conductor se enfrenta a un mundo que en cierta medida es "incierto": no sabe si el camino estará cortado, si habrá baches en la ruta (y a causa de estos puede dañarse el automóvil), algunas veces podrá confundirse de camino y en tal caso el GPS deberá re-calcular, etc. Similarmente, el director técnico de un equipo de futbol puede diseñar una estrategia basada principalmente en el estilo de juego de su adversario, pero una vez iniciado el juego las cosas pueden no salir de acuerdo a lo planeado. En tal caso, el técnico deberá modificar su estrategia inicial. En cualquiera de los ejemplos mencionados, las intervenciones no sólo son ex-ante, sino también continuas o sistemáticas. Y lo sistemático no es producto de la incapacidad del interventor, sino de que éste se enfrenta a un escenario abierto e incierto, donde a fin de cuentas apostará por seguir un determinado camino, pero reconociendo que en el mismo podrán aparecer contingencias que induzcan al interventor a hacer correcciones.

\section{CONSIDERACIONES FINALES}

Cartwright asevera las regularidades o conjunciones constantes de eventos son producto del ejercicio de máquinas nomológicas. Se ha visto que éstas resultan ser invaluables para satisfacer propósitos intervencionistas, ya que su ejercicio garantiza la producción del resultado predicho en el modelo. Esta concepción puede ser de utilidad en las ciencias naturales, donde las capacidades son efectivamente estables, pero no lo es tanto en las sociales. En los sistemas naturales, las "actividades" (MACHAMER: DARDEN; CRAVER, 2000) emergen de las propiedades de las entidades participantes. 
En los sistemas sociales, en cambio, las actividades son las propias acciones de las personas. Dichas actividades dependen de una miríada de factores, muchos de ellos de carácter subjetivo (como por ejemplo el modo en que las personas interpretan las señales del mundo o en cómo éstas forman sus expectativas). Si bien no es imposible que en algunas ocasiones se observe una conducta uniforme, el enfoque de Cartwright no es congruente con la lógica de los fenómenos sociales. A lo largo del presente trabajo se ha intentado mostrar que se logra una mayor adecuación introduciendo las nociones de "árboles de posibilidades" o "resultados de final abierto". Una consecuencia casi inmediata de este cambio es concebir a los modelos de máquinas nomológicas no como representaciones de factores causales aislados, sino como anteproyectos de sistemas complejos para alcanzar resultados en el mundo real. Ahora bien, a diferencia de los enfoques manipulabilistas tradicionales, las intervenciones no deberían ser pensadas únicamente en términos "ex-ante", sino también en términos de sistematicidad o de continuidad.

IVAROLA, Leonardo. Nomological machines, models and intervention: their problems in the social realm. Trans/form/ação, Marília, v. 39, n. 2, p. 101-118, Abr./Jun., 2016.

\begin{abstract}
In the present paper, a critique of Nancy Cartwright's nomological machines approach in the social sciences will be made. It is argued that social phenomena do not correspond to the logic of "capacities", but to the logic of "possibility trees" or "open-ended results". Stability or invariance, although possible in the social realm, seem to be more the exception than the rule. This has important implications for the purposes of intervention and control. According to traditional manipulabilistic accounts, invariance is essential for achieving any interventionist purpose, because it allows predicting the result of a manipulation with a high degree of confidence. However, in this paper it will be shown that even though the obtaining of invariant knowledge is not entirely plausible in the social realm, interventions may be effective. To do this, it will be necessary to replace the "ex-ante" notion of intervention with another notion that involves continuity. It will also be crucial to take into account the clauses or conditions specified in models, since such models play the role of being blueprints for generating results.
\end{abstract}

KEYWORDS: Nomological machines, intervention, open-ended results, social capacities. 


\section{REFERENCIAS}

BUNGE, M. La relación entre la sociología y la filosofia. Madrid: EDAF, 2000.

CARTWRIGHT, N. Nature's capacities and their measurement. Oxford: Clarendon Press, 1989. 294, 1995.

Ceteris Paribus Laws and socio-economic machines. Monist, v. 78, n. 3, p. 276-

Models: the blueprints for laws. Philosophy of Science: Proceedings of the 1996 Biennial Meetings of the Philosophy of Science Association. Part II: Symposia Papers, v. 64, Suppl. p. S292-S303, 1997.

. Capacities. In: DAVIS, J.; HANDS, W.; MÄKI, U. (Ed.). The handbook of economic methodology. Cheltenham: Edward Elgar, 1998. p. $45-48$.

. The Dappled world. Cambridge: Cambridge University Press, 1999a.

. The Vanity of Rigour in economics: theoretical models and Galilean Experiments. Centre for Philosophy of Natural and Social Science. Discussion. 1999b. (También publicado en: CARTWRIGHT, N. Hunting causes and using them: approaches in philosophy and economics. Cambridge: Cambridge University Press, 2007a, p. 217-261).

. Hunting causes and using Them: approaches in philosophy and economics. Cambridge: Cambridge University Press, 2007b.

. If no capacities then no credible worlds. But can models reveal capacities? Erkenntnis, v. 70, n.1, p. 45-58, 2009a.

. Causal laws, policy predictions, and the need for genuine powers. In: HANDFIELD, T. (Ed.). Dispositions and causes. Oxford University Press, 2009b. p. 127157.

. Presidential address: will this policy work for you?: predicting effectiveness better: how philosophy helps. Philosophy of Science, v. 79, n. 5, p. 973-989, 2012.

.; HARDIE, J. Evidence-based policy. a practical guide to Doing It Better. Oxford: Oxford University Press, 2013.

DOW, S. The methodology of macroeconomic Thought. Cheltenham: Edward Elgar, 1998.

HÉDOIN, C. Models in economics are not (Always) nomological machines: a pragmatic approach to economists' modelling practices. Philosophy of the Social Sciences, v. 20, n. 10, p. 1-36, 2012.

HENDRY, D. Causality and exogeneity in non-stationary time series. In: Causality: metaphysics and methods. Technical Report, Centre for Philosophy of Natural and Social Science, London School of Economics, CTR 18-04, 2004.

KEYNES, J. La teoría general de la ocupación, el Interés y el dinero. Buenos Aires: Fondo de Cultura Económica, 2001. (Original publicado em 1936). 
LAWSON, T. Economics and reality. London: Routledge, 1997. Reorienting economics. London: Routledge, 2003.

MACHAMER, P.; DARDEN, L.; CRAVER, C. Thinking about mechanisms. Philosophy of Science, v. 67, n. 1, p. 1-25, 2000.

MÄKI, U. On the method of idealization in economics. Poznan Studies in the Philosophy of the Sciences and the Humanities, v. 26, p. 319-354, 1992.

. Models are experiments, experiments are models. Journal of Economic Methodology, v. 12, n. 2, p. 303-315, 2005.

. Missing the world: models as isolations and credible surrogate systems. Erkenntnis, v. 70, n. 1, p. 29-43, 2009.

. Models and the locus of their truth. Synthese, v. 180, n. 1, p 47-63, 2011.

MITCHELL, S. Pragmatic laws. Philosophy of Science, v. 64, n. 4, p. S468-S479, 1997.

Press, 2003.

Biological complexity and integrative pluralism. Cambridge: Cambridge University

REISS, J. Do we need mechanisms in the social sciences?. Philosophy of the Social Sciences, v. 37, n. 2, p. 163-184, 2007.

WOODWARD, J. Explanation and invariance in the special sciences. British Journal for the Philosophy of Science, v. 51, n. 2, p. 197-254, 2000.

. What is a mechanism? A counterfactual account. Philosophy of Science, v. 69, p. S366-S377, 2002.

Making things happen: a theory of causal explanation. Oxford: Oxford University Press, 2003.

Recebido em 28/10/2015

Aceito em 18/02/2016 
IVAROLA, L. 